\title{
MARKSHEET VERIFICATION USING BLOCKCHAIN
}

\author{
NORA NAIK ${ }^{1}$, DATTARAM NAIK ${ }^{2}$, ROHIT NAIK ${ }^{3}$, ASHVEDHPEDNEKAR $^{4}$, \\ SHAUNAKVALAWALKAR ${ }^{5} \&$ VINAYAK SINAI BHANDIYE ${ }^{6}$ \\ ${ }^{l}$ Assistant Professor, Department of Computer Engineering, Agnel Institute of Technology \& Design, Goa, India \\ ${ }^{2,3,4,5,6}$ Students, Department of Computer Engineering, Agnel Institute of Technology \& Design, Goa, India
}

\begin{abstract}
The blockchain is an incorruptible digital ledger of economic transactions that can be programmed to record not just financial transactions but virtually anything of value. Blockchain techniques maintain transparency of data on all its peers on the network. This technique can be applied in various fields. The blockchain technique is considered to be very secure in terms of protecting data. Each block in a blockchain consist of hash value of the previous block and a ledger that is stored redundantly across may nodes. Once the data is recorded in the blockchain it becomes difficult to change that data. In the present system of marksheet verification in order to check the originality of marksheet the company needs to contact the concerned institution/university to validate the marksheet. In this regard lot of time and human resources are wasted to accomplish this process. In the proposed system using blockchain, we are making marksheet verification easier and secure so that nobody can temper with the marksheet once the mark sheet is stored on the blockchain. Also the marksheet can be verified by comparing the hash value to the one stored in the blockchain.
\end{abstract}

KEYWORDS: Blockchain, Marksheet, Verification Network \& Data

Received: May 23, 2019; Accepted: Jun 12, 2019; Published: Jul 04, 2019; Paper Id.: IJCSEITRDEC20196

\section{INTRODUCTION}

Before blockchain was used in crypto currency, particularly in the domain of cryptography and data structure. The hash tree was the primitive form of blockchain, also known as Merkle Tree. This data structure functioned by verifying and handling data between computer systems. The validation of data was important to make sure nothing was changed or altered and also ensure that false data was not send during transmission in a peer to peer network of computers. It is used to maintain and prove the integrity of data being shared. Blockchain would contain a secure history of data exchanges and utilize peer to peer network to timestamp and verify its exchange. Blockchain is a decentralized database that records the data, like finance contracts, physical assets and supply chain information. It has acquired a successful recognition over time and used by Bitcoin.

The two phrases of blockchain are: Block and Chain. Block is the original entity that documents the time stamp and is encrypted. By use of a private key it is governed by an owner. All the distributed chains are updated on the real-time basis in case of edits introduced. It is the most active part of the blockchain that records the previous transactions. On completion, the block moves back to the blockchain and the new one is generated which forms the chains that are linked to one another. The new developed block gets connected to the previous block. The complete history of the users and addresses are contained in the blockchain. The copy of the data cannot be regenerated while distribution of data. While making a transaction, anyone can access the chain since it is open and distributed and it is not governed by any other third party or the organization. In blockchain we can view the data or information but cannot 
control it.

\section{FEATURES OF BLOCKCHAIN}

The main features of blockchain technology are:

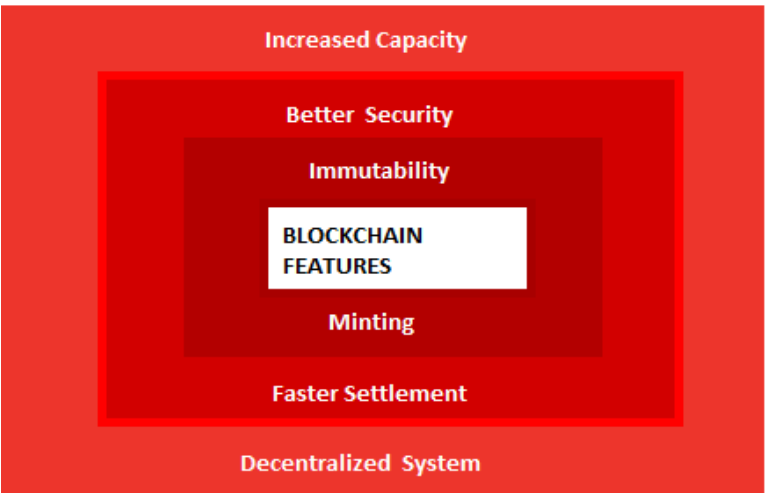

Figure 1: Features of Blockchain

\section{Increased Capacity}

It increases the capacity of the whole network, since there are lot of computers working together which offers a great power in total then few of the devices where the things are centralized.

\section{Better Security}

There is no chance of shutting down of the system since Blockchain technology has a better security. The main reason is that the Blockchain network is secured by a number of computers called nodes and transaction on this network is confirmed by these nodes.

\section{Immutability}

One of the main values of Blockchain is creating immutable ledgers. The databases which are centralized are most likely to get hacked and to keep these databases secure they require trust in the third party.

\section{Faster Settlement}

A lot of settlement time is required, which usually takes days to proceed may cause the traditional banking systems to slow down. Because of this reason these banking institutes needs to upgrade their banking systems. With the help of Blockchain technology we can solve this problem as it can settle money transfer at really fast speeds. It provides convenience to the consumers since it saves a lot of time and money from these institutions.

\section{Decentralized System}

The decentralized technology gives the power to store assets in a network which further accesses by the means of the internet. An asset can be like a document, a contract, etc. By this the owner can have a direct control over his account which gives the owner a power to transfer his assets to anyone he wants to by the means of a Key that is linked to his account.

\section{Minting}

By help of the Blockchain we can solve the various ways of minting a problem of manipulation. The role of the miner is to create a block for many new methods which has also been introduced as proof of work is the approach by which an 
individual can prove that he is engaged in a significant amount of computation work.

\section{Blockchain Technology Types}

- Permissionless Blockchain, anyone can create an address and start communicating with the blockchain. An example of permissionless blockchain is Internet, where anyone can create or design a website and interact with the world by creating an address on the network.

- Permissioned Blockchain is the private blockchain specially designed to allow an organization to exchange information and transactions efficiently. In this type of blockchain each member is well defined.

\section{WORKING OF BLOCKCHAIN}

Figure 2 shows how a Blockchain works.

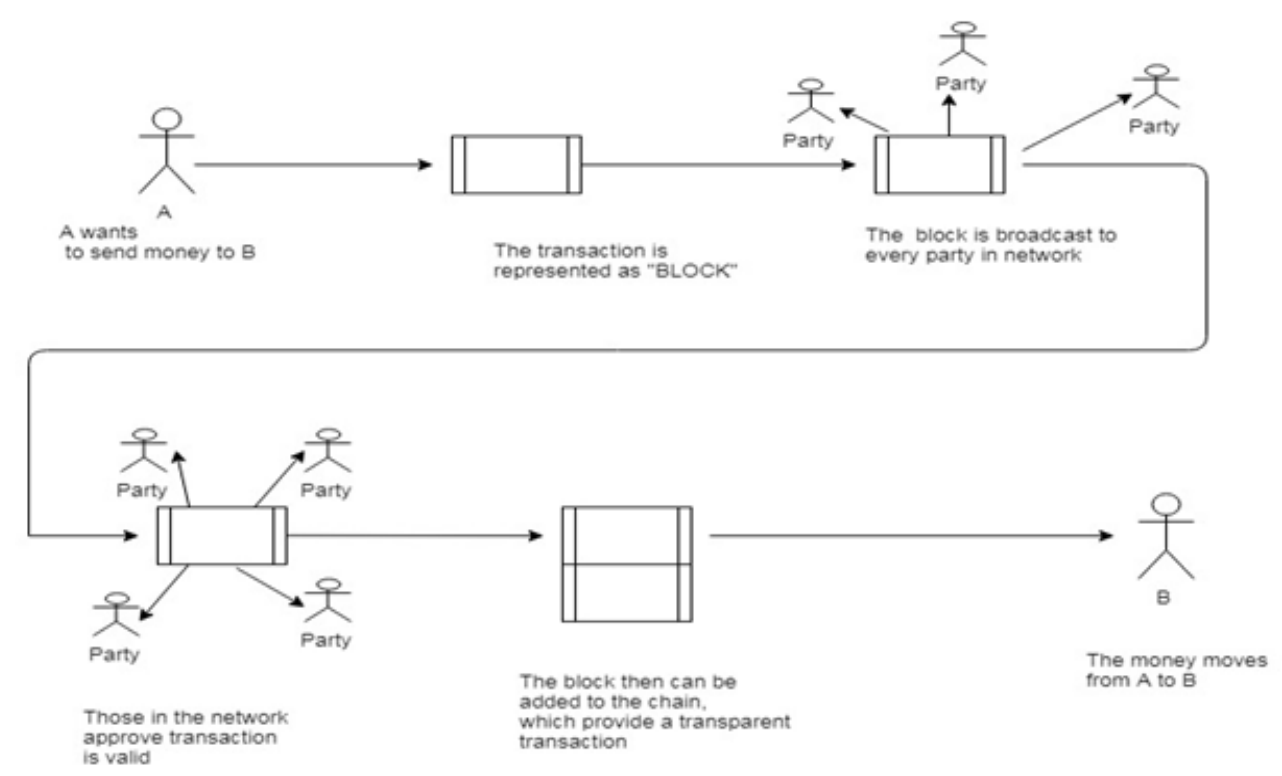

Figure 2: Blockchain Flow Diagram

\section{Parameters of a Block}

Block header field consists of version, last block, transactions, time, target.

- Version: It describes the structure of data inside a block.

- Last Block: It is an identification number for the previous block.

- Time: States the current time.

- Target: A value that reduces work by adding candidate block to the blockchain.

\section{PROPOSED SYSTEM}

\section{Marksheet Verification using Blockchain Technology}

The process of verification of marksheets is made much easier by implementing a blockchain based approach. In addition to making the verification process easier, the use of blockchain also helps in eliminating counterfeiting. The transparency of the blockchain model makes it nearly impossible to have a counterfeit certificate. The proposed system deals 
with marksheet verification within a single department of our institute. The features of the proposed system are

- Privacy: The marks of a student will not be disclosed.

- Accuracy: The marks will be stored accurately within the system.

- $\quad$ Robustness: Nobody will be able to modify the marksheet details but only be able to verify it.

\section{Flow of Project}

The flow of the proposed system is as follows in figure 3 below

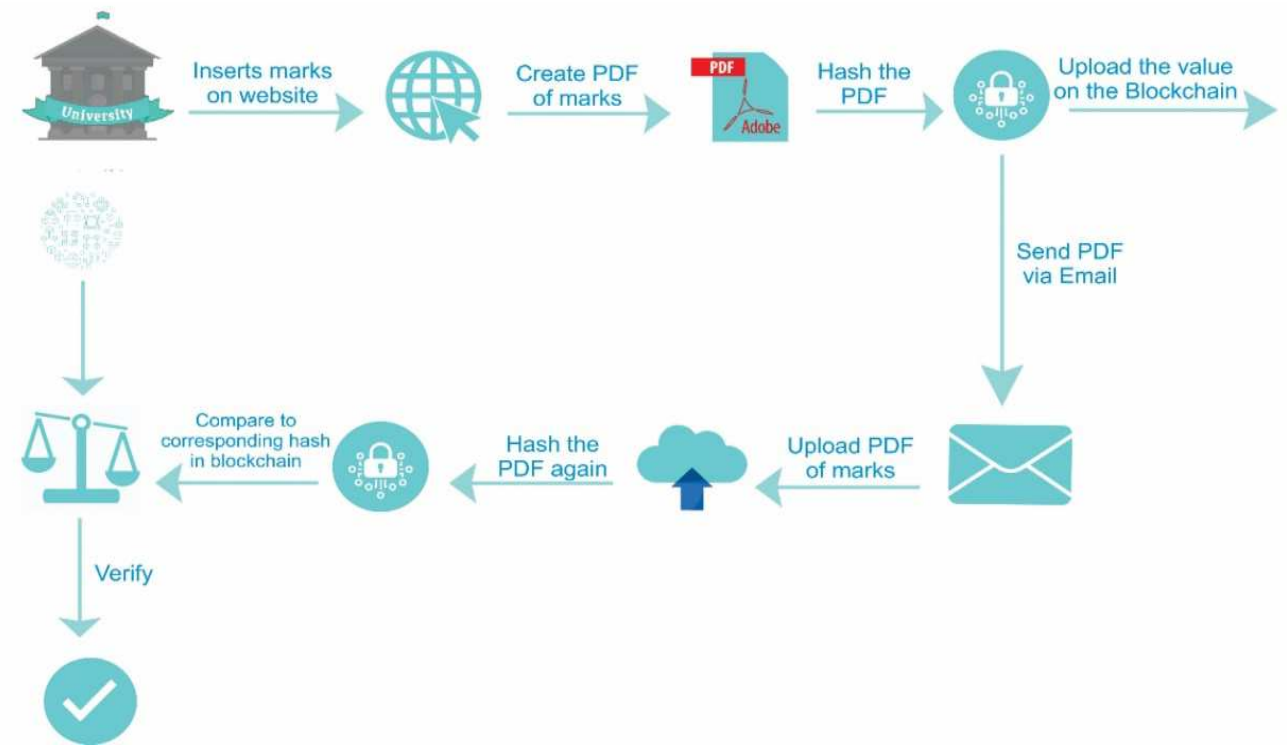

Figure 3: Flow of the Proposed System

As show in figure 3 above, the proposed system has two distinct sections one concerning the issuing of the certificate and the other concerning the verifying of the certificate.

\section{Issuing Certificate}

The steps performed to issue a marksheet certificate is as follows:

- Enter marks of students-Here the admin enters the marks of the student on to the institute's designed website.

- PDF Generation-Using these marks and student details entered, a PDF of the marksheet is created.

- Hashing- The PDF is hashed. Hashing can be done using one of the many hashing algorithms. In the proposed system SHA256 is used to compute the hash value.

- Upload to blockchain- Hash value that is generated in step iii above is stored on the blockchain after hashing it with the private key.

- Transmit to student- Marksheet is then sent to student along with the institute public key.

\section{Certificate Verification}

The generated PDF can then be sent by the student to whoever they wish to send it to along with the public key of the institute. The following steps are then performed for certificate verification: 
- Upload marksheet- Once the receiver gets the marksheet they can verify it by going to the website and uploading the PDF they received.

- Calculating hash value-The PDF is then taken and its hash value is calculated using the Roll number of student as the key.

- Comparison to stored value- Receiver then compares the value to the hash of the corresponding marksheet in the blockchain and if the hash value is the same then the marksheet is authentic and verified, else it is not.

\section{IMPLEMENTATION OF THE PROPOSED SYSTEM}

\section{Home Page}

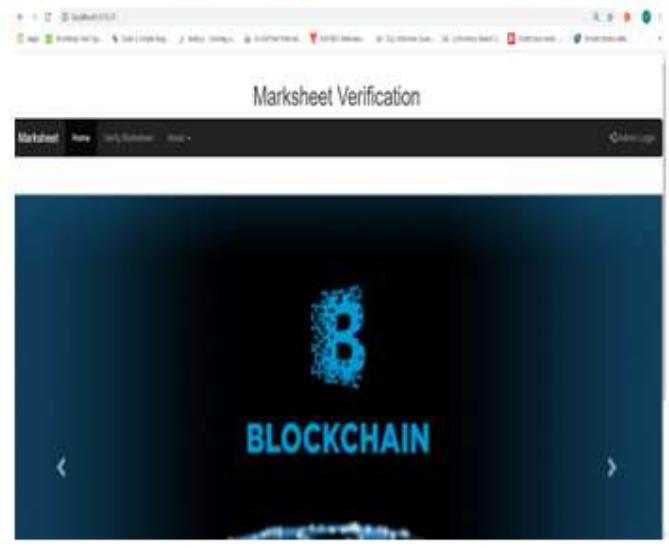

Figure 4: Home Page

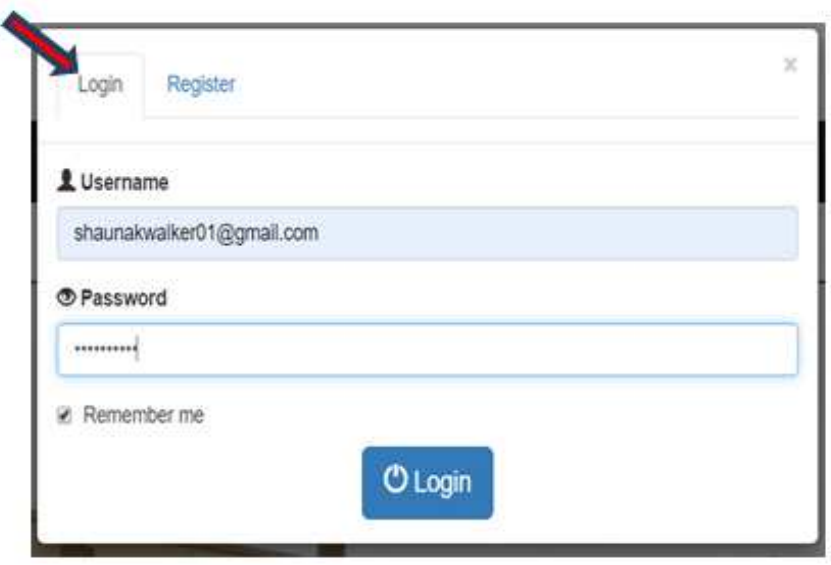

Figure 5: Admin Login Page

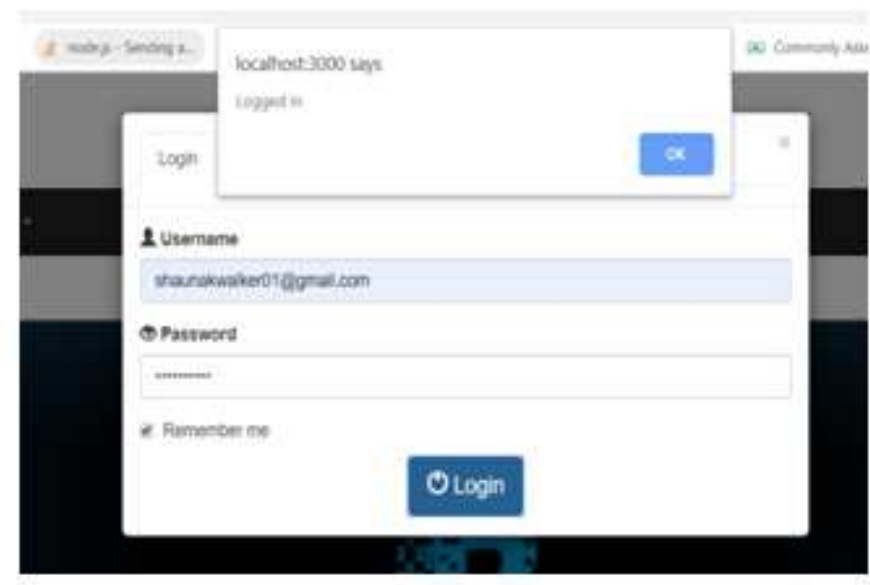

Figure 6: Logged in Successfully Message

Admin

Creating Marksheet from Admin Side Select the Semester and Enter the marks accordingly 

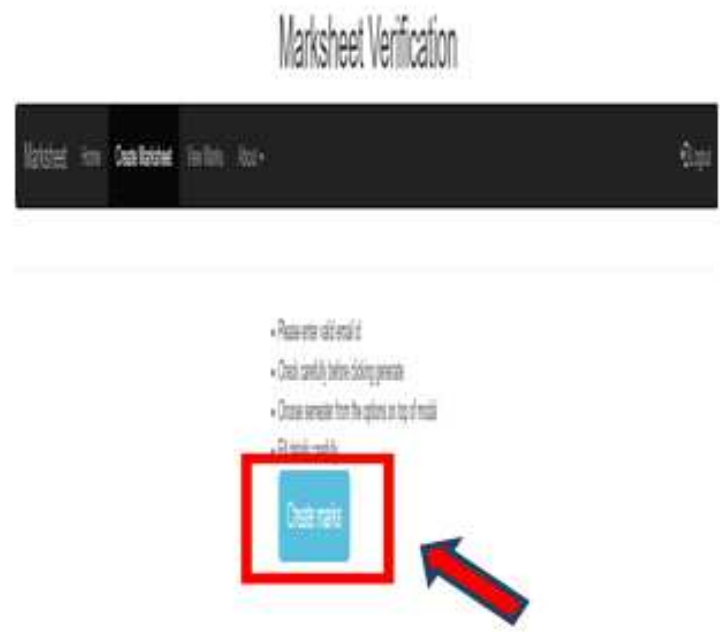

Figure 7: Create New Marksheet

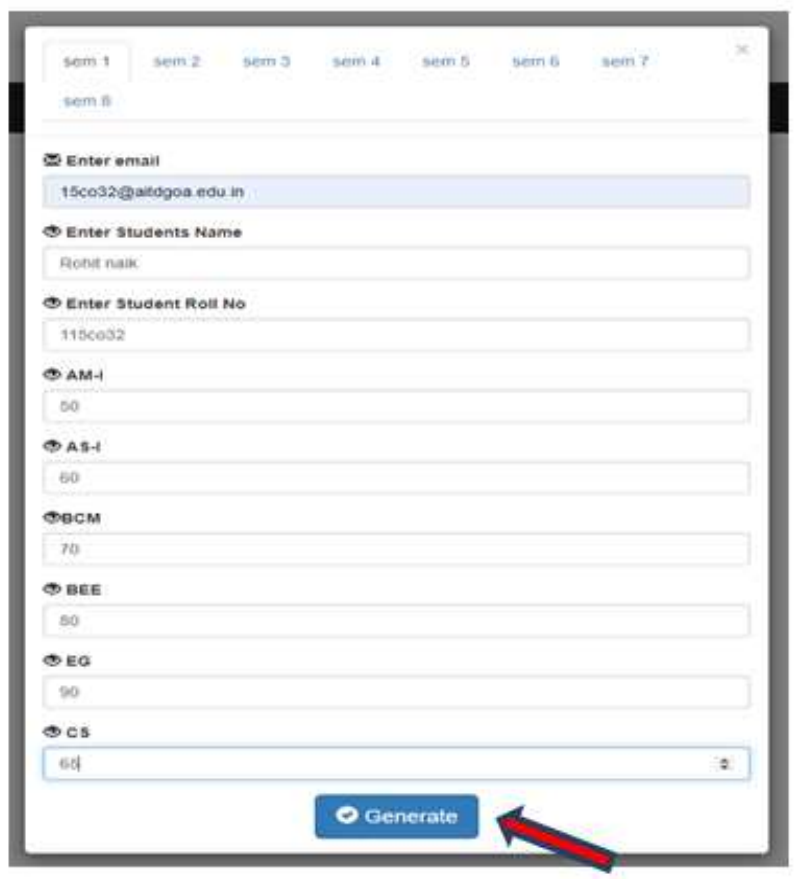

Figure 8: Enter the Necessary

Once the 'Generate' button on the webpage is pressed, hash value is generated and is entered into the blockchain.

The pdf file containing the result of the student corresponding to the generated hash value is sent to them by email.

c:|Users| Vinayak |Docunents / websitebackup node website.js

Server runing at Port 3000

Database is connected

Entering in db

1150032

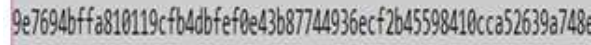

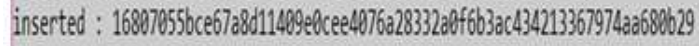

Entered in do

Enail sent

Figure 9: Hash Value of the Marksheet

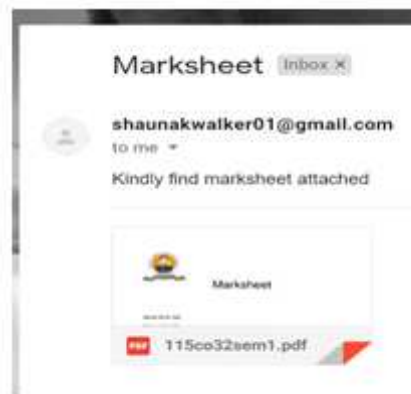

Figure 11: The Pdf Containing the Student's Result is Received in the Mai

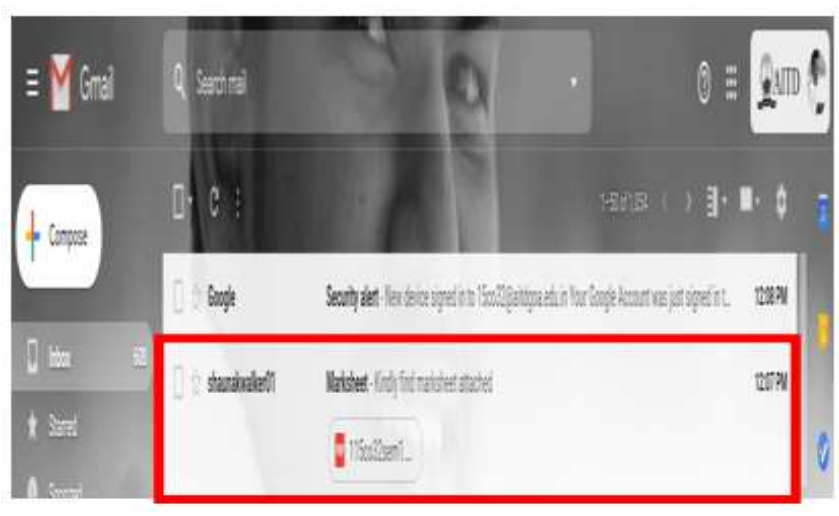

Figure 10: The Above Image Shows that the Mail is Received by the Appropriate Student

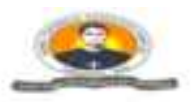

Marksheet

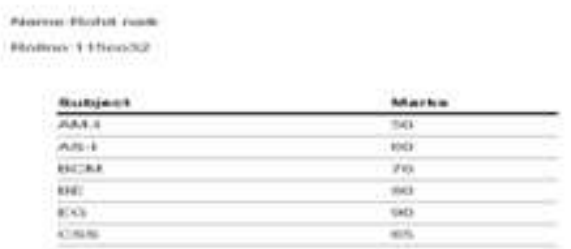

Figure 12: The Image Shows Above the Marksheet of a Student 
After Figure 12, Log Out from admin side.

\section{Client Side}

In case the client wishes to verify the result received by them, then they can do so as follows. In the interface shown below, the client must put their company name, student's name, upload the marksheet they have received and enter the key, i.e. the name of the file to be uploaded.

Click on the marksheet verification tab on 'navigation bar'

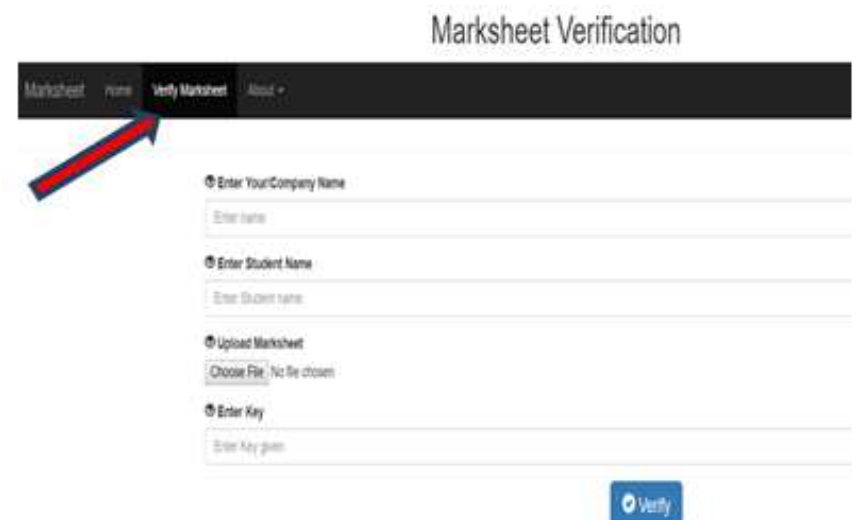

Figure 13: Verify Marksheet
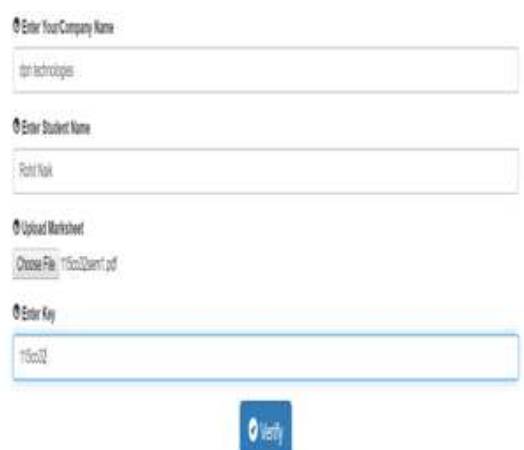

Figure 14: Fill in the Details

On click of "Choose File" button, the client uploads the file from the client's system as shown below

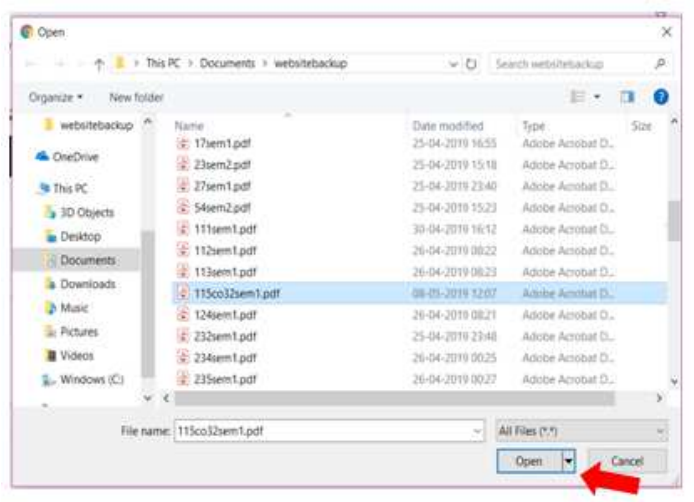

Figure 15: Choose Marksheet

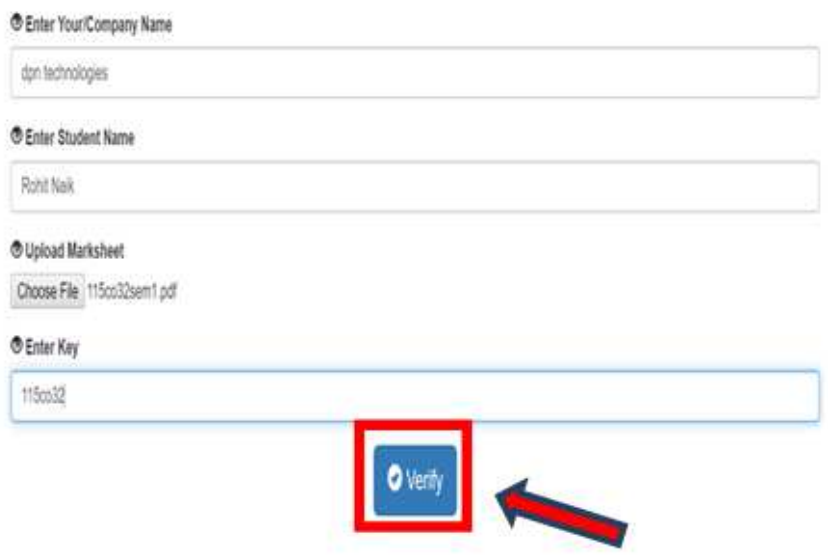

Figure 16: Verify

After the client sends the marksheet for verification, whether it is modified or not is verified and displayed. 


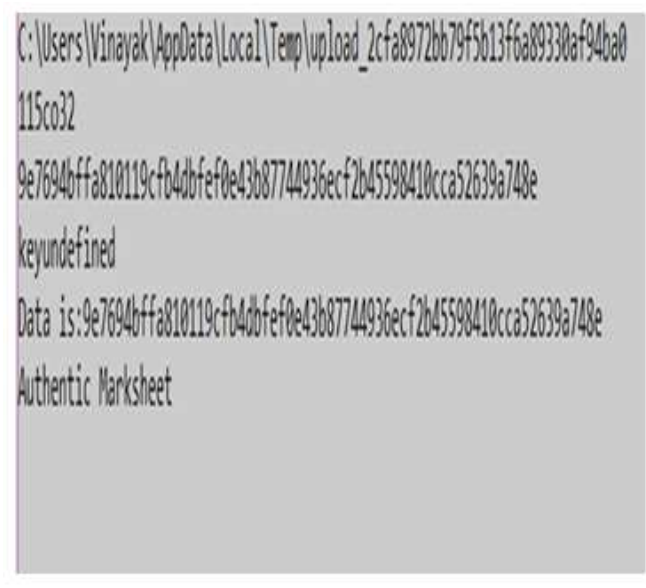

Figure 17: Admin Side
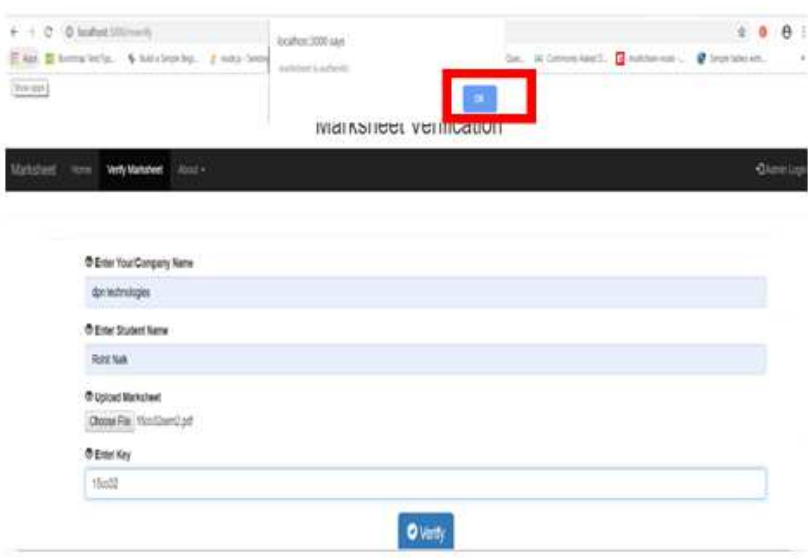

Figure 18: The Alert Box Shows whether Certificate is Authenticate or Not

\section{CONCLUSIONS}

In the older systems of marksheet verification chances of misuse of data was high. This is eliminated by using a blockchain based approach in the proposed system of marksheet verification. This approach overcomes quite a number of issues associated with older marksheet verification processes. It reduces certificate forgeries and also ensures that the marksheets are secure, valid as well as confidential. Furthermore, the proposed system offers many benefits both to the issuing institution as well as the recipients of the marksheets. It increases the efficiency in terms of security of data and storage. Additionally, to verify a certificate the end user need not contact the university as all the data required to validate the certificate is present on the blockchain itself. All that has to be done at the user end is generate the hash value and compare it to the stored hash value. This also greatly reduces the time required for certificate verification

In future, the system for marksheet verification can be modified by using smart contracts. Smart contracts are like digital protocols which are used to enforce a contract without any control from a third party. Smart contracts help to automatically compute the results eliminating illegal activities completely

\section{REFERENCES}

1. R. L. Rivest, A. Shamir, L. Adleman, A method for obtaining digital signatures and public-key cryptosystems, Communications of the ACM, v.21 n.2, p.120-126, Feb. 1978

2. A. Ouaddah, A. A. Elkalam, and A. A. Ouahman, "Towards a novel privacy-preserving access control model based on blockchain technology in IoT," in Europe and MENA Cooperation Advances in Information and Communication Technologies. Springer, 2017, pp. 523- 533 .

3. J. van Beusekom, F. Shafait, and T. M. Breuel, "Text-line examination for document forgery detection," Int. J. Doc. Anal. Recognit., vol. 16, no. 2, pp. 189-207, 2013.

4. M. Singh and D. Garg, “Choosing best hashing strategies and hash functions," Int. Advance Computing Conf., 2009, pp. 50 - 55

5. Samit Shivadekar (IT, SIES GST, Navi Mumbai, India,) Stephen Raj Abraham (IT, SIES GST, Navi Mumbai, India,) Sheikh Khalid (IT, SIES GST, Navi Mumbai, India), "Document Validation and Verification System", International Journal of Advanced Research in Computer Engineering \& Technology (IJARCET) Volume 5 Issue 3, March 2016

6. Anoukh. N, "Block Chain System for Data Management", International Journal of Financial Management (IJFM) Vol. 08, Issue 1, pp. 1-6, Jan 2019 
7. Neethu Gopal and Vani V Prakash, "Survey on Blockchain Based Digital Certificate System”, International Research Journal of Engineering and Technology (IRJET) e-ISSN: 2395-0056 Volume: 05 Issue: 11 | Nov 2018 www.irjet.net p-ISSN: 2395-0072

8. S. Balasubramanian, R. Prashanthlye, and S. Ravishankar, "Mark sheet verification," 2009 3rd Int. Conf. Anti-counterfeiting, Secure. Identify. Commun. ASID 2009, 2009.

9. P. Schmidt, “Certificates, Reputation, and the Blockchain,” MIT Media Lab, 2015.

10. Multichain: https://www.multichain.com/developers/

11. Blockchain: To Be or not to be an article by Mr. Ganesh Faterpekar:

12. https://medium.com/@ganeshfaterpekar/blockchain-to-be-or-not-to-be-748710bee4f9 
